\title{
La investigación biográfico-narrativa: una vía para comprender las trayectorias profesionales de los docentes de nuevo ingreso
}

Mtra. en Inv. de la Edu. Blanca Estela Martínez Victoria

blanquisisis@ outlook.es

Instituto Superior de Ciencias de la Educación del Estado de México

Recibido: 27 de abril 2020

Aceptado: 25 de mayo del 2020

\section{Resumen}

Este artículo se propone repensar a la investigación biográfico narrativa como un enfoque propio y no como una metodología que se adiciona a las ya existentes. En este sentido, hace posible que emerja a través del discurso de las personas únicas y diferentes que habitan en el refugio de sí y para sí a partir de las narrativas individuales la ocurrencia de sucesos pequeños pero auténticos de los cuales dan cuenta los textos que pueden ser leídos e interpretados revelándose en sus discursos sus historias de vida. Es así como tal enfoque permite emprender la búsqueda de los sentidos y significados, mismos que edifican en torno a la práctica docente aquellos profesionales formados en áreas afines a las asignaturas del nivel que se incorporan a la tarea docente, sin haber cursado una trayectoria de formación escolar normalista o de la Universidad Pedagógica Nacional (UPN) para tal efecto. El argumento principal se plantea a partir del conocimiento de los vínculos que se establecen entre la trayectoria escolar y la trayectoria profesional; primordialmente encaminada a la búsqueda de sentidos y significados que se construyen en ésta última. Así, se presenta un avance en cuanto al marco teórico y metodológico para adentrarse en la búsqueda de las significaciones desde la perspectiva de Bolívar, Domingo y Fernández (2001) como vía para la recuperación de los relatos de los sujetos y desde la perspectiva hermenéutica de Ricoeur (1990) como posibilidad de hallar los sentidos y significados que asignan a las acciones, los participantes en la investigación.

Palabras clave: Trayectoria profesional, textos, interpretación. 


\title{
Biographical-narrative research: a way to understand the professional careers of new teachers
}

\begin{abstract}
This article aims to rethink narrative biographical research as its own approach and not as a methodology that is added to those that already exist. In this sense, it enables the ocurrence of small but authentic events of which the text that can account for emerge from the discourse of the unique and different people who live in the refuge of themselves and for themselves from the individual narratives. Be read and interpreted revealing their life stories in their speeches. Thus, such an approach allows the search for the senses and meanings to be undertaken, which are built around the teaching practice by those professionals trained in areas related to the subjects of the level curriculum that are incorporated into the teaching task, without having completed a career normalistic school training or the National Pedagogical University (UPN) for this purpose. The main argument arises from the knowledge of the links established between the school career and professional career; primarily aimed at the search for meanings and meanings that are built on the latter. Thus, an advence is presented regarding the theoretical and methodological framework to delve into the search for meanings from the perspective of Bolívar, Domingo and Fernández (2001) as a way to recover the stories of the subjects and from the hermeneutical perspective of Ricoeur (1990) as a possibility to find the meanings assigned to the actions, the participants in the research.
\end{abstract}

Keywords: Professional career, texts, interpretation

\section{Introducción}

El artículo es producto del trabajo de investigación intitulado: De profesionistas a docentes de la escuela secundaria en el marco de la Ley General del Servicio Profesional Docente. Sentidos y significados, como parte de mi formación doctoral en el Instituto Superior de Ciencias de la Educación del Estado de México.

Una vez realizado el estado del arte, se pone de manifiesto que hasta ahora no se ha investigado acerca de los sentidos y significados de los docentes que han ingresado a la escuela secundaria en el marco de la Ley General del Servicio Profesional Docente, siendo aquellos quienes se han enfrentado a un examen de oposición para tener acceso a la docencia contando 
con una preparación profesional o perfil profesional afín, como se señaló en la convocatoria pública y abierta estableciendo las pautas de participación para los profesionistas que tienen pretensiones de incursionar en las actividades docentes. Hecho que no es un asunto de menor importancia, sobre todo para quienes se desempeñan en este campo. Por lo cual se determina que se hace necesaria la investigación de tal fenómeno, dado que, de acuerdo con Ricardo Sánchez Puentes (1993). Existe una laguna en torno a él.

Para el presente escrito se tiene dos objetivos, en primero de ellos es argumentar el uso de la investigación biográfico narrativa como método y su uso para comprender las trayectorias profesionales de aquellos profesionistas que, sin ser profesores de carrera, realizaron un examen de oposición y obtuvieron una plaza en educación secundaria. El segundo pretende abrir la discusión en torno a la relevancia de la investigación biográfico-narrativa para el conocimiento de las trayectorias de los docentes de manera específica.

De lo anterior se desprende una pregunta que guía el escrito ¿cómo podemos acercarnos al conocimiento de las trayectorias profesionales que construyen los docentes de nuevo ingreso a la educación secundaria? Ubica entonces como categorías centrales a la práctica docente, trayectoria académica, trayectoria profesional, sentidos, significados, desde referentes teóricos que se consideran acordes.

Se desarrolla en el marco del paradigma cualitativo con el enfoque metodológico de la investigación biográfico-narrativa en el ámbito de la educación planteada por Bolívar, Domingo y Segovia (2001), en la perspectiva de interpretación hermenéutica desde Paul Ricoeur (1990). Adicionalmente se hizo uso de estrategias para el acopio de información en el marco de la recuperación de relatos y narrativas autobiográficas, primordialmente la entrevista y los escritos autobiográficos privilegiando la oralidad como la fuente más importante de la investigación, que permitan ir dando solidez a la investigación consolidándose en la tesis de grado. 
El artículo en su estructura consta de seis apartados, el primero de ellos refiere a la forma en que han ingresado los profesionistas a la docencia, el segundo aborda la vida como punto de partida de la investigación biográfico narrativa, en el tercero se señala el marco categorial de la investigación, en cuarto lugar, se alude a la investigación narrativa en educación en tanto su vigencia y relevancia; para finalmente tratar la perspectiva hermenéutica en la investigación narrativa. Enseguida se cierra temporalmente el artículo a la vez que da apertura a nuevas ideas y construcciones en torno al tema aquí planteado.

\section{La condición de ingreso a la profesión docente}

A partir de la reforma al artículo $3^{\circ}$ constitucional el 26 de febrero de 2013, de acuerdo con la publicación realizada en el diario Diario Oficial de la Federación (DOF), se ha dado origen a una de las denominadas leyes secundarias, siendo ésta; la Ley General del Servicio Profesional Docente (LGSPD), misma que en el Capítulo III Artículo 21 señala:

El Ingreso al Servicio en la Educación Básica y Media Superior que imparta el Estado y sus Organismos Descentralizados, se llevará a cabo mediante concursos de oposición, preferentemente anuales, que garanticen la idoneidad de los conocimientos y las capacidades necesarias, con sujeción a los términos y criterios (DOF, 2013, p. 12).

Entonces, la acreditación de un examen de conocimientos otorga a los aspirantes de ingreso a la actividad docente la condición de idóneos con la cual parecería que ejercer la docencia es una tarea sencilla que cualquiera que cumpla con los requisitos puede desarrollar siempre y cuando adquiera el estatus de idoneidad de cual hace mención la LGSPD.

Sin embargo para el ejercicio docente se precisa de elementos básicos para el desarrollo de actividades de aula, de entre los cuales se pueden destacar las habilidades para realizar la planificación didáctica, el conocimiento de estrategias para interactuar con los alumnos haciendo posible el trabajo grupal, la incorporación pertinente de recursos didácticos que medien el aprendizaje de los alumnos, la integración de propuestas y/o estrategias de evaluación que den cuenta de los niveles de desarrollo alcanzados por los alumnos respecto del aprendizaje, el 
conocimiento de la psicología del desarrollo infantil. Sin que por ello la actividad docente se reduzca a un acto mecánico y reproduccionista.

Los profesionistas no formados en docencia se supone que pudieran carecer de ello, a diferencia de quienes se formaron para tal efecto, ya que estos últimos han tenido un contacto con la práctica docente y sus implicaciones desde su trayectoria formativa por lo que tienen conocimiento de los elementos antes señalados.

En este orden de ideas la precisión de la LGSPD se apareja con lo que los propios organismos internacionales asumen como uno de los temas centrales de la política educativa. Entre ellas se pueden leer dos que enuncian: "El maestro es el factor más importante del sistema educativo" y "la calidad del sistema educativo está en función de la calidad de sus maestros" (Aceves, 2015, p. 40). Hasta aquí parecería considerarse, que la perspectiva era contratar a los egresados de las escuelas formadoras de docentes, sin embargo, se percibe en la reorientación de los instrumentos de evaluación una consideración distinta al leer que:

Se puso énfasis en que la evaluación para el Concurso de Plazas no era una acreditación de los conocimientos adquiridos en las escuelas normales, sino más bien garantizar que el docente por contratar tuviera el dominio de los nuevos contenidos curriculares, conocimiento indispensable para el óptimo desarrollo de su labor docente (Aceves, 2015, p. 42).

A decir de la autora, de ahí en adelante, los cambios continuaron, desde la modificación de áreas a evaluar, la cantidad de reactivos, el tiempo de resolución del examen y la elaboración de pruebas específicas para cada asignatura, hasta la aceptación de problemáticas a las que se enfrenta el servicio profesional docente, como el contratar a docentes que obtienen en realidad puntajes bajos en la evaluación y por supuesto el desvanecimiento o desdibujamiento de la pedagogía y la didáctica como elementos esenciales para la actividad docente.

El ingreso al servicio profesional pareciera tener como objetivo "contratar al que garantice el dominio de conocimientos y competencias básicas para la enseñanza" (Aceves, 2015, p. 45), lo cual nos lleva a plantear una interrogante, ¿se tiene la certeza de que la aprobación de un examen, 
es garantía de tales conocimientos y competencias?, esto ¿alcanza para ser un docente en la práctica?, los docentes de nuevo ingreso nos pueden contar su verdad en torno a ello.

\section{La vida como punto de partida de la investigación}

Es en los principios que señala Hoyos (2000), para orientar la construcción de la investigación que se enuncian como finalidad, coherencia, fidelidad, integración y comprensión; donde puede verse de alguna manera reflejada la vida como prevalencia de historias singulares, de subjetividades y de contextos cada uno con sus particularidades y que sin duda son el eje la investigación narrativa desde la constitución de relatos. Y en estos la articulación de la vida y su concurrencia en el espacio áulico para la conformación de las trayectorias profesionales, como es el caso de los docentes de nuevo ingreso.

Al situarnos en este fenómeno específico se acerca "reducir el campo de observación a un tipo particular de trayectoria o de contexto" (Bertaux, 2005, p. 20), reducción entendida como la delimitación para circunscribirse en un contexto, así tal recorte permite ver con mayor claridad un problema, para situarlo en el entendido de que un fenómeno de cualquier tipo, solo adquiere sentido en el marco de la totalidad de acuerdo con Kosik (1967).

En este sentido, el ámbito de lo educativo es justamente la dimensión donde se ubica el hecho motivo de la indagatoria por lo cual es preciso reconocer el distanciamiento entre quien indaga y el objeto de la indagatoria. "El distanciamiento es definido como la capacidad del científico de diferenciarse emocionalmente (incluyendo la ideología) de los objetos de estudio, observarlos críticamente y alcanzar un conocimiento racional" (Manrique, Di Matteo, \& Sánchez Trousel, 2016, p. 989), donde sin duda la observación crítica lleve a quien investiga, a la construcción de un conocimiento racional, recuperando la singularidad de cada uno como sujetos que se constituyen a partir del establecimiento de la interacción con los otros.

Sobre todo, cuando desde la perspectiva del Banco Mundial, se puede leer a Hanushek, Piopiunik y Wiederhold (2018), que los docentes son considerados el elemento más importante y valioso para la construcción de futuros exitosos en países que se han atrevido a profesionalizar la carrera docente. 


\section{Marco categorial de la investigación}

En la idea de que todo pensamiento parte de un marco teórico para interpretar la realidad, y de que éste se va modificando debido a que nunca logramos asir la realidad porque no es posible llegar al saber absoluto, se recuperan las categorías primordiales para ser abordadas.

La primera será enmarcada en el ámbito de lo social como señala Berteaux (2005): docentes de nuevo ingreso, a quienes se les define de acuerdo con la LGSPD en su Capítulo I, Artículo $4^{\circ}$ Fracción XXV, como el profesional en la educación secundaria "que asume ante el Estado y la sociedad la responsabilidad del aprendizaje de los alumnos en la Escuela y, en consecuencia, es responsable del proceso de enseñanza aprendizaje, promotor, coordinador, facilitador, investigador y agente directo del proceso educativo" (DOF, 2013, p. 4) y que por supuesto ha sido sujeto a un proceso de selección para el acceso al Servicio Profesional Docente.

Como segunda categoría teórica, la idea de trayectoria, partiendo desde el concepto acuñado en el ámbito de la Física, pero traída al espacio de la investigación, se puede citar a Serrano y Ramos, para quienes trayectoria, “...se opone a las clásicas formas de realizar biografías, autobiografías, mismas que regularmente aceptan la segmentación del sentido de la vida en forma secuencial, cronológica o como evolución” (2011, p. 12), por tanto aquí también se recupera la idea de Bourdieu, citada por dichos autores en cuanto a la trayectoria "como serie de posiciones sucesivamente ocupadas por un mismo agente (o un mismo grupo) en un espacio en sí mismo en movimiento y sometido a incesantes transformaciones" (2011, p. 12-13), transformaciones que se asocian con las actividades que de manera cotidiana se desarrollan en un campo en el cual se encuentran inmersos los docentes, refiriendo su contexto de acción en el cual desarrollan la práctica docente.

La tercera de las categorías a recuperar es el sentido, el cual para Holzapfel (2005), "ante todo nos cobija y ello en especial debido al vínculo que tenemos con algo" (p. 47), de tal forma que, siguiendo al autor, pareciera ser que el sentido justifica lo que hacemos. Pero sobre todo otorga la razón o razones por las cuales hacemos lo que hacemos y como lo hacemos según señala Gilles Deleuze, quien considera que sentido, es todo lo que se da como pensamiento, es 
una maquinaria singular que es elaborada y diría quien ahora escribe incluso reelaborada siempre de nuevo; misma que permite, como en este caso; adentrarse al terreno en que se lleva a cabo la práctica profesional de los docentes de nuevo ingreso para conocer que una cosa en sí es lo que es y no otra para ellos.

Una cuarta categoría y no menos importante resulta ser la práctica docente de los profesionistas de nuevo ingreso a la docencia. Para ello se recupera por ahora, de Cecilia Fierro el concepto de práctica docente como:

Praxis social, objetiva e intencional en la que intervienen los significados, las percepciones y las acciones de los agentes implicados en el proceso -maestros, alumnos, autoridades educativas y padres de familia-, así como los aspectos políticoinstitucionales administrativos y normativos que, según el proyecto educativo de cada país, delimitan la función del maestro" (Fierro \& Lesvia, 1999, p. 21).

Cuando se habla de profesionistas es porque se hace referencia a quienes cuentan con un grado académico de licenciatura afín a las áreas o asignaturas de la currícula de educación secundaria, y no refiere a quienes han tenido una formación para la docencia. Sin negar que el grado de licenciatura sea afín, tanto para los que se formaron para la docencia como para aquellos cuya formación fue (si no ajena a la docencia), sí en una disciplina o área específica y diferenciada del ejercicio docente. Sin que por ello se niegue a los docentes el rango de profesionistas, simplemente, es para establecer la distinción para referirse a unos u otros.

Finalmente significados, es una categoría no menos importante, se enmarca en el propio enfoque narrativo y desde la ciencia interpretativa que busca justamente encontrar tales significados y que se encuentran implícitos en los hechos humanos siendo "motivaciones, sentimientos, deseos, propósitos que no pueden ser expresados en definiciones, enunciados factuales o definiciones abstractas" (Bolívar A. , 2002, p. 6), podría ser entonces un elemento del propio lenguaje que pretende comunicar, en palabras de Ricoeur (1995), es la intención referente del hablante.

\section{La investigación narrativa en educación. Su vigencia y relevancia}

Justo con este antecedente es que se plantea la apuesta por la investigación narrativa, en la cual los informantes siempre hablan de ellos mismos, de otra manera, se estaría negando e incluso 
eliminando su propia individualidad, esto resulta un hecho reconocido por la investigación narrativa que se presenta como:

Método de investigación e interpretación en el que no cabe la posibilidad de generalización. Se alista dentro de una metodología de corte hermenéutico, que permite conjuntamente dar significado y comprender las dimensiones cognitivas, afectivas y de acción. Contar las propias vivencias, y leer (en el sentido de interpretar) dichos hechos/acciones, a la luz de las historias que los agentes narran, se convierten en una perspectiva peculiar de la investigación (Bolívar, Domingo, \& Fernández, 2001, p. 10).

La búsqueda de las cualidades se hará básicamente a partir de las entrevistas, así como los escritos autobiográficos, puntualizando que "la oralidad (entrevistas, historias, conversaciones, etc.) es la fuente más importante de la investigación biográfica, no excluye otros medios escritos” (Bolívar, Domingo, \& Fernández, 2001, p. 156)

El desarrollo de la investigación en el ámbito educativo no es la excepción, en este campo se requiere de lo anteriormente señalado, bajo el entendido de que las interacciones sociales son justamente aquellas que producen hechos, sucesos o fenómenos derivados de la interacción social que se establece entre los diferentes agentes del sistema educativo. Para el caso específico de esta investigación y de manera primordial, los docentes de nuevo ingreso. Sin olvidar que dichas interacciones sociales no han de reducirse a lo objetivo, lo posible entonces es tomar el mundo puramente y de forma totalmente exclusiva, desde las condiciones de quienes son los sujetos de la investigación, recuperando sus cualidades.

\section{La perspectiva hermenéutica en la investigación narrativa}

Para efecto de acceder a la comprensión en torno a la ocurrencia de fenómenos en el acontecer social, nos situamos especialmente en el análisis de aspectos fundamentales, como son en primer lugar, el lenguaje; sobre todo cuando recordamos que lo que pretendemos interpretar son los sentidos y significados que construyen/atribuyen los docentes de nuevo ingreso a la práctica docente, desde las múltiples vivencias, no a las personas mismas, sino a las narraciones hechas texto, donde el lenguaje prevalece por el hecho de que los seres mismos son lenguaje. 
Habrá que recordar nuevamente que la búsqueda importante del significado se ha de centrar en el relato, una vez que ha sido convertido en texto, entonces: "El significado del texto no está atrás del texto, sino enfrente de él; no es algo oculto sino algo develado lo que tiene que ser entendido no es la situación inicial del discurso, sino lo que apunta hacia un mundo posible gracias a la referencia del texto" (Ricoeur P. , 1995, p. 100). Bajo esta premisa, se entiende un texto siguiendo su movimiento desde el significado a la referencia: es decir en el doble sentido desde lo que se dice y desde aquello de lo que se habla, implicando incluso desde Schleiermacher (1999), una suerte artística y sensibilidad creativa, asociada al desarrollo de la narración que realiza el investigador acerca del relato de los participantes en la investigación, en cuya trama se encuentra el secreto de la acción. Sin que esto represente un camino lineal para el investigador, sino más bien circular.

Para el autor, la actividad de comprensión tiene ocurrencia cuando existe la disputa entre la verdad y la explicación. "Tal debate comienza en un simple análisis sobre nuestra manera de pensar y de hablar sobre las cosas, pero que, por el movimiento del argumento, se dirige a las cosas mismas que requieren nuestras concepciones acerca de ellas mismas" (Ricoeur P., 2002, p. 149). Bajo la mirada del autor citado, se ha de dar la oportunidad de recuperar sustancialmente la vida de las personas desde el conocimiento y análisis de sus trayectorias relatadas y convertidas en textos, ante todo cuando lo que se trata de recuperar elementos para ser interpretados.

Cabe puntualizar que la hermenéutica se define como el arte basado en la interpretación de textos, y desde la perspectiva filosófica que plantea Ricoeur (1990), constituye un procedimiento que permite expresar la habilidad interpretativa desde la personal y específica historicidad. Esta interpretación, también ha de recurrir al conocimiento de las condiciones históricas para su propia comprensión en la compleja red que no determina, sino que va constituyendo de manera progresiva a los sujetos pensados desde la incompletud siempre o bien, desde la finitud de la condición humana.

Así, en los planteamientos de Ricoeur, la hermenéutica surge en los textos que pueden ser pensados como la posibilidad de atrapar los discursos y/o relatos a través de la escritura. Un relato que se encuentra directamente ligado con quien lo habla en primera instancia, pero que posteriormente adquiere un carácter hasta cierto punto de independencia cuando se plasma en un 
texto y que es seguido ahora por otro que lo lee para incursionar mediante ello en un mundo posible que representa una realidad para el otro, ahora desde la tarea que ha de desarrollar quien pretende interpretarlo llevando a cabo la tarea hermenéutica.

Lo importante en la tarea interpretativa para el investigador, sin duda será tener toda la apertura posible para dejarse decir algo por el texto mismo, además de recuperar y extraer no lo que se encuentra de manera literal en el texto sino llevar a efecto una reelaboración del texto por parte del intérprete desde su propia mirada, sin perder de vista la esencia que imprimió al texto, quien hizo el relato.

Desde luego que resulta particularmente importante recordar que "lo que exige ser interpretado en un texto es su sentido, y el acto de su apropiación es más una fusión del mundo del lector y del mundo del texto que una apropiación del intérprete sobre el texto" (Ricouer. 2004 , p. 20). Por supuesto que se hace necesario pensar que la tarea hermenéutica no es una cuestión simple, más bien resulta una tarea compleja porque se halla estrechamente ligada a lo ontológico, sin embargo, sí representa un reto que puede y debe ser afrontado y enfrentado por el investigador para encontrar los sentidos y significados.

Es imprescindible además pensar que para el ejercicio interpretativo hay que considerar al relato como "la trama, el medio privilegiado para esclarecer la experiencia temporal inherente a la ontología del ser-en-el-mundo" (Ricoeur. 2004, p. 26). Mismo que una vez plasmado en el texto, nos hace reflexionar en torno a que ha sido producido desde contextos específicos, desde subjetividades heterogéneas, desde modos de ser y de concebir el mundo y la vida, a partir de referentes

\section{Consideraciones finales}

Puede señalarse que el avance hasta el momento, en la investigación permite acercamiento en la clarificación de las categorías teóricas que hacen posible la comprensión interpretación, mediante el establecimiento de la vigilancia epistemológica que orienta el alcance de los objetivos planteados en esta investigación, desde el proceder metodológico en los planos de la investigación. Es decir, el marco teórico y metodológico como vía posible para acercarse a la interpretación de los sentidos y significados que los docentes atribuyen en torno a un ejercicio 
docente quizá impensado desde la trayectoria formativa, sin embargo, vivenciado en la trayectoria docente que la cual se construyen.

\section{Referencias bibliográficas}

Aceves, A. M. (2015). La evaluación docente en México. En G. Guevara, La evaluación docente en México (págs. 40-63). México: Fondo de Cultura Económica INEE.

Bertaux, D. (2005). Los relatos de vida. Perspectiva etnosociológica. Barcelona, España: Ediciones Bellaterra.

Bolívar, A. (2002). "De nobis ipsis silemus?": Epistemología de la investigación biográficonarrativa en educación. . Revista Electrónica de Investigación Educativa, 1-26.

Bolívar, A., Domingo, \& Fernández. (2001). La investigación biográfico-narrativa en educación. Enfoque y Metodología. Madrid: La Muralla.

DOF. (11 de 09 de 2013). Decreto por el que se expide la Ley General del Servicio Profesional Docente. Diario Oficial de la Federación.

F., S. (1999). Los discursos sobre hermenéutica. Cuadernos anuario filosófico . España: Universidad de Pamplona.

Fierro, C., y Lesvia, F. B. (1999). Transformando la práctica docente. México: Paidós.

Holzapfel, C. (2005). A la búsqueda del sentido. Santiago de Chile: Editorial Sudamericana.

Hoyos, B. C. (2000). Un modelo para la investigación documental: Guía teórica-práctica construcción de Estados del Arte. Medellín, Colombia: Señal Editora.

Husserl, E. (2008). La crisis de las ciencias europeas y la fenomenología trascendental. Buenos Aires, Argentina : Prometeo Libros.

Kosík, K. (1967). Diáléctica de lo concreto. Estudio sobre los problemas del hombre y el mundo. México: Grijalbo S. A. . 
Manrique, M. S., Di Matteo, M. F., y Sánchez Trousel, L. (2016). Análisis de la implicación: construcción del sujeto y del objeto de investigación. Cadernos de pesquisa, 984-1008.

Ricoeur, P. (1990). Freud: Una interpretación de la cultura. México D. F.: Siglo XXI editores.

Ricoeur, P. (1995). Teoría de la interpretación. Discurso y excedente de sentido. Mexico D. F. : Siglo XXI Editores.

Ricoeur, P. (2002). Del texto a la acción. México: Fondo de Cultura Económica.

Ricoeur, P. (2004). Tiempo y narración I. Configuración del tiempo en el relato histórico. Buenos Aires Argentina: Siglo XXI Editores.

Serrano, C. J. (2011). Trayectorias: biografías y prácticas. México: Horizontes Educativos. 